\title{
ESTRATÉGIAS DE MEMÓRIA UTILIZADAS POR IDOSOS PARA LEMBRAREM DO USO DOS SEUS MEDICAMENTOS
}

\author{
Maria Cristina Werlang 1 \\ Irani Iracema de Lima Argimon² \\ Lilian Milnitsky Stein ${ }^{3}$
}

\section{Resumo}

Os múltiplos tratamentos requeridos para tratar as patologias que comumente surgem durante o envelhecimento acarretam em prescrições geralmente complexas e difíceis de seguir. Isto pode ainda ser agravado pelo freqüente declínio cognitivo observado nesse período, sendo o esquecimento uma das causas que torna os idosos fortes candidatos a não aderir à prescrição médica. Estudos têm demonstrado que o emprego de estratégias de memória prospectiva por pacientes idosos pode contribuir para uma maior adesão à terapêutica. Aqui, buscou-se identificar o emprego de estratégias de memória por idosos para lembrarem do uso de seus medicamentos. A amostra foi de 53 idosos com até setenta anos, não-institucionalizados e independentes para exercerem suas atividades diárias, e que utilizavam no mínimo três medicamentos de uso contínuo. Adaptou-se à nossa língua e realidade um questionário para identificar as estratégias de memória utilizadas para lembrar do uso dos medicamentos. A adesão à prescrição médica foi examinada através de auto-relato. Os instrumentos foram aplicados através de entrevista estruturada e individual. Os resultados indicaram elevado índice de uso de estratégias internas retrospectivas e alta taxa de adesão $(73,6 \%)$ auto-relatada, o que reforça achados prévios sobre a tendência dos idosos em superestimar sua adesão à prescrição. $\mathrm{O}$ uso de estratégias de memória pode minimizar ou compensar as dificuldades mnemônicas observadas em idosos, como as enfrentadas para seguir o regime terapêutico, auxiliando na preservação da sua autonomia e promoção da sua qualidade de vida.

Palavras-chave: Memória prospectiva. Estratégias de memória. Aderência à prescrição. Idosos.

\footnotetext{
1 Farmacêutica, Doutora em Gerontologia Biomédica. Pontifícia Universidade Católica do Rio Grande do Sul. E-mail: crisw.ez@terra.com.br

2 Psicóloga, Doutora em Psicologia. Pontifícia Universidade Católica do Rio Grande do Sul. E-mail: argimoni@ pucrs.br

3 Psicóloga. PHD em Psicologia. Pontifícia Universidade Católica do Rio Grande do Sul. E-mail: lilian@ pucrs.br
}

Estud. interdiscip. envelhec., Porto Alegre, v. 13, n. 1, p. 95-115, 2008. 


\section{Introdução}

O processo de envelhecimento é individual e multifatorial, contemplando aspectos biológicos, sociais, intelectuais, econômicos e funcionais (JECKEL; CUNHA, 2002). Embora seja difícil definir um limite de idade entre o indivíduo adulto e o idoso, o critério cronológico é empregado com o propósito de estabelecer limites norteadores, sendo adotado o parâmetro de sessenta anos para os países em desenvolvimento (PAPALÉO-NETTO, 2002). O envelhecimento pode ser acompanhado tanto de alterações decorrentes de modificações fisiológicas, como também daquelas que surgem com maior freqüência com o passar dos anos, tais como a hipertensão, o diabetes mellitus e as neoplasias. Segundo Culos-Reed et al. (2000), cerca de $85 \%$ dos idosos têm pelo menos uma doença crônica que requer tratamento farmacológico. De acordo com dados de uma pesquisa sobre padrões de vida na terceira idade, os problemas crônicos de saúde verificados nos idosos gaúchos com maior freqüência foram: hipertensão $(26,4 \%)$, osteoporose $(17,8 \%)$, doença cardíaca (16,8\%) e diabetes (11,9\%) (RIO GRANDE DO SUL, 1997).

Flores e Mengue (2005) avaliaram o perfil de consumo de medicamentos por idosos residentes no Rio Grande do Sul. Os autores encontraram que $91 \%$ da amostra utilizavam, em média, 3,2 medicamentos, sendo que a classe terapêutica mais utilizada foi a de fármacos para o sistema cardiovascular. O multiuso de medicamentos em idosos também foi relatado por outros pesquisadores, tais como Rozenfeld (2003) e Chen, Dewey e Avery (2001). Contudo, a medicalização no idoso deve ser bem monitorada, porque se encontra associada a diversos problemas, dentre eles a falta de adesão à prescrição médica (LEITE; VASCONCELLOS, 2003). Segundo Cipolle, Strand e Morley (2004), esta é compreendida como o não-seguimento do paciente à sua prescrição médica, podendo, no entanto, ser comprometida pelo freqüente quadro de declínio cognitivo e limitações físicas evidenciados nas pessoas idosas (ROZENFELD, 2003; WORLD. . ., 2001).

A não-adesão ao regime farmacológico pode ocorrer de forma intencional ou não. Quando está relacionada a limitações físicas, aspecto financeiro e cognitivo é considerado não-intencional. Dentre os aspectos cognitivos, encontram-se as dificuldades de compreensão e recordação das orientações recebidas e horários de administração dos medicamentos pelos pacientes. A literatura na área tem apontado que as causas mais freqüentes de falta de adesão dos idosos concentram-se em motivos não-intencionais. Por exemplo, Lowry et al. (2005), observaram que entre $40 \%$ dos pacientes considerados não-aderentes, $31 \%$ foram decorrentes de causas não-intencionais. 
A taxa de adesão à prescrição médica em idosos foi estudada por Roth e Ivey (2005) por meio de um instrumento de auto-relato. Os dados encontrados indicaram que 53\% dos entrevistados eram "não-aderentes". Entretanto, há que se considerar que os pacientes tendem a superestimar sua adesão quando empregados os métodos de auto-relato, mas que, por questões de custo e praticidade, são os mais utilizados (MOSCATI; PERSANO; CASTRO, 2000).

As questões relativas ao ato de recordar são usualmente divididas em dois tipos de memória: a retrospectiva e a prospectiva. Quando uma pessoa relembra informações aprendidas no passado, está executando uma tarefa de memória retrospectiva, e quando relembra a execução de uma ação que será desenvolvida no futuro, refere-se à memória prospectiva (GUYNN; McDANIEL; EINSTEIN, 1998; EINSTEIN et al., 2005).

Conforme postulam Park e Kidder (1996), a memória prospectiva envolve a recuperação de uma intenção de ação que está armazenada na memória de longo prazo, requerendo um relembrar retrospectivo. Neste sentido, Einstein et al. (1997) conceituaram o seguimento do regime terapêutico prescrito como uma tarefa de memória prospectiva. Relembrar o contexto da informação e seu detalhamento refere-se ao componente retrospectivo, e recordar de fazer a ação orientada no tempo exato se refere à memória prospectiva (GUYNN; McDANIEL; EINSTEIN, 1998; EINSTEIN et al., 2005). Park e Hall (2000), como também McDaniel e Einstein (2000) e Einstein et al. (2005), observaram em seus estudos que os idosos apresentaram dificuldades no desempenho de tarefas de memória prospectiva, sugerindo que a memória para tarefas a serem desempenhadas no futuro é menos automática e requerem maior empenho do que as tarefas de memória retrospectiva.

Park e Hall (2000) referem ainda que, para usar um medicamento corretamente, as pessoas utilizam um componente prospectivo e um componente retrospectivo. $\mathrm{O}$ primeiro componente refere-se ao resgate mnemônico para execução de uma ação futura, enquanto o segundo abrange recordar as instruções relativas ao plano terapêutico.

Devido ao declínio cognitivo mais pronunciadamente após os 70 anos, muitos idosos podem não possuir recursos suficientes para a função adequada dos aspectos retrospectivos da adesão ao medicamento (PARK; HALL, 2000). Este fato está alinhado com a hipótese formulada anteriormente por Einstein e McDaniel (1996), segundo a qual o deficit da idade sobre tarefas de memória prospectiva é resultante de uma pobre função da memória retrospectiva. Posteriormente, McDaniel e Einstein (2000) postularam 
que a memória prospectiva envolve mecanismos dependentes de múltiplas variáveis, as quais influenciam no processo de recordar e no tipo de estratégia de memória a ser selecionada para um bom desempenho mnemônico. Segundo Einstein et al. (2005), as estratégias de memória podem auxiliar tanto na compreensão da informação quanto na sua retenção.

As estratégias de memória são classificadas em internas ou externas, segundo o mecanismo pelo qual a informação é manipulada. As primeiras envolvem atividades mentais, tais como codificação mnemônica e estratégias de recuperação de memória; e as externas contemplam as manipulações externas do ambiente, sendo estas as mais utilizadas pelos idosos para tarefas de memória prospectiva. Especificamente, no caso do uso de medicamentos, ao associar os horários de administração dos fármacos com atividades rotineiras, os pacientes estarão utilizando uma estratégia de memória interna. E, ao fazerem anotações e deixarem bilhetes em locais visíveis para lembrarem de usar os seus medicamentos, estarão usando uma estratégia externa. Independentemente da natureza interna ou externa da estratégia a ser escolhida, quando as pessoas buscam essas ferramentas por si próprias, mostram-se mais motivadas para o uso das mesmas, tornando-se mais convictas da sua efetividade. Entretanto, certas estratégias externas têm sua eficácia diminuída com o passar do tempo (GOULD; DIXON, 1997).

As tarefas de memória prospectiva podem ainda ser baseadas no evento ou no tempo, embora em idosos as segundas apresentem maior prejuízo mnemônico porque podem interferir no aspecto prospectivo da administração medicamentosa. Os medicamentos são prescritos para serem administrados num esquema específico de horários, com intervalos de tempo definidos, como, por exemplo, três vezes ao dia, ou de quatro em quatro horas. Todavia, este esquema possui desvantagens para as pessoas idosas, uma vez que este processo requer grande demanda de auto-iniciativa (SMITH, 2003). No entanto, algumas pesquisas demonstraram que os idosos podem transformar as tarefas baseadas no tempo naquelas baseadas no evento, ao associá-las com algo que seja feito rotineiramente, como, por exemplo, administrar o medicamento antes de assistir a um programa específico na televisão (HICKS; MARSH; COOK, 2005; SMITH, 2003).

McDaniel e Einstein (2000) descobriram que a memória prospectiva de idosos para tarefas rotineiras tem um grande potencial para criar confusões em relação a ações que foram ou não executadas. Segundo os autores, a idade e a divisão da atenção contribuem para erros de repetição e omissão quando, por exemplo, um idoso administra em caráter não-intencional duas doses seguidas do seu medicamento. Já no caso dos erros de omissão, 
verifica-se o esquecimento do uso de algum medicamento prescrito pelo médico. Num outro experimento, West e Craik (2001) relacionaram o deficit de desempenho da memória prospectiva de idosos ao aumento de ocorrência de erros de omissão cometidos por estas pessoas. Neste contexto, o desenvolvimento de métodos de treinamento mnemônico e de estratégias que auxiliem no recordar prospectivo pode contribuir positivamente para um melhor desempenho das atividades diárias por idosos (PARK; MAYHORN, 2000), como é o caso do uso de medicamentos.

Os idosos tendem a usar estratégias de memória como um mecanismo compensatório para o esquecimento dos medicamentos a serem administrados. Spiers e Kutzik (1995) encontraram que os idosos preferem usar estratégias externas, associando o medicamento a algo rotineiro, o que também foi apontado por Branin (2001), com exceção do fato de sugerir que estas estratégias estariam associadas a mecanismos mnemônicos internos. Segundo o mesmo autor, depressão e ansiedade foram preditores do tipo de estratégia utilizada.

Determinadas estratégias podem ser utilizadas na promoção da adesão medicamentosa pelo paciente, tais como as caixas para acondicionamento diário dos comprimidos, anotações, calendários, seleção prévia dos medicamentos que devem ser tomados diariamente (GENUA et al., 2002), cartelas de comprimidos com calendários (PAULÓS et al., 2005) e uso de despertador e esquemas mentais (BRANIN, 2001; McDONALD-MISZCZAK et al., 2004). Alguns autores já avaliaram a adesão à prescrição médica em idosos e comprovaram que estes pacientes têm maior dificuldade de recordar as instruções médicas do que os adultos jovens (BROWN; PARK, 2002; PARK; HALL, 2000). No entanto, a utilização de estratégias de memória como uma alternativa de promoção da saúde, no sentido de auxiliar o paciente idoso a seguir seu regime terapêutico, ainda foi pouco estudada.

A partir dessas considerações, o presente estudo buscou identificar a freqüência do tipo (retrospectivo e prospectivo) e natureza (interna e externa) das estratégias de memória utilizadas por idosos para lembrarem do uso dos seus medicamentos. Também foram investigadas as freqüências de uso das estratégias de memória em relação a variáveis sócio-demográficas e terapêuticas. Para tanto, adaptou-se um instrumento de avaliação das estratégias de memória utilizadas por idosos para lembrar do uso de medicamentos (GOULD; McDONALD-MISCZACK; KING, 1997). 


\section{Método}

Foi conduzido um estudo transversal para buscar descrever os tipos e a natureza das estratégias de memória utilizadas por idosos para lembrar do uso de medicamentos, e também foram investigadas algumas variáveis sócio-demográficas relativas à terapêutica medicamentosa.

\subsection{Participantes}

Foram definidas como variáveis de inclusão adultos com idade entre sessenta e setenta anos, alfabetizados, que utilizavam um mínimo de três medicamentos de uso contínuo, que não possuíam monitoramento clínicodomiciliar nem de profissionais de saúde, e que eram independentes para as suas atividades da vida diária.

A amostra foi constituída por 53 idosos de ambos os sexos, na referida faixa etária, procedentes de duas origens:

a) programa de pesquisa de um hospital privado de Porto Alegre, desenvolvido no município de Gravataí, cuja população-alvo era de um nível sócio-econômico baixo;

b) posto de saúde de um hospital público de Porto Alegre que atende a pessoas de nível sócio-econômico médio-baixo.

O limite de idade até setenta anos foi estabelecido com base nos estudos de Park et al. (1992), que evidenciaram uma maior deficiência cognitiva em idosos a partir de 71 anos.

O projeto do presente estudo e o termo de consentimento livre foram submetidos aos Comitês de Ética em Pesquisa e aprovados conforme ofícios $n^{\circ}$ 159/00, em 13 de julho de 2000, e no 29/00, em 9 de agosto de 2000, respectivamente. Toda a pesquisa foi realizada segundo a Resolução 196/96 (BRASIL, 1996) que regulamenta a ética em pesquisa no Brasil.

\subsection{Instrumentos}

Foram utilizados os seguintes instrumentos: Ficha Sócio-demográfica; Ficha Farmacoterapêutica, que buscava identificar o nome dos medicamentos utilizados e sua classe terapêutica; Inventário de Independência para Atividades da Vida Diária (AVD) (KATZ et al., 1963); Auto-relato da adesão à prescrição médica (MORISKY; GREEN; LEVINE, 1986); e Questionário de Memória Prospectiva (PMMQ) (GOULD; McDONALD-MISCZACK; KING, 1997), o qual foi submetido a um processo de adaptação. 
O processo de adaptação do PMMQ envolveu as seguintes etapas: tradução para o português por dois juízes que dominam ambos os idiomas; parecer de médico geriatra e farmacêutico com conhecimento na área em questão para verificar sua aplicabilidade no contexto brasileiro, e estudo prépiloto com cinco sujeitos selecionados de acordo com os mesmos critérios empregados para compor a amostra definitiva, a fim de avaliar a compreensão do instrumento. Após procedidas as modificações necessárias, esta versão do PMMQ foi submetida a uma testagem-piloto com mais dez idosos, sendo que, a partir desta última, foi verificada a necessidade de exclusão de mais duas questões. As alterações no instrumento e sua aplicação no presente estudo foram aprovadas pela autora do instrumento por meio de correio eletrônico. A versão final do PMMQ adaptado ficou composta por um total de 23 perguntas, ficando assim distribuídas: seis itens para o componente externo-prospectivo, quatro itens para o componente externo-retrospectivo, oito itens para o componente interno-prospectivo, quatro itens para o componente interno-prospectivo, permanecendo a última questão aberta relativa a qualquer estratégia que fosse utilizada pelo sujeito (Apêndice). A ordem de apresentação das questões foi estabelecida de forma aleatória.

As respostas eram marcadas em uma escala Likert ( 0 a 3), a qual foi apresentada por meio de um cartão ilustrativo, contendo as alternativas: nunca (0), quase nunca (1), quase sempre (2) e sempre (3).

\subsection{Procedimentos}

As entrevistas foram realizadas individualmente pela pesquisadora e por três acadêmicos de Farmácia, treinados para fins de uniformização de linguagem. Foram selecionados todos os participantes que apresentavam os critérios de inclusão na amostra. O convite deu-se por contato telefônico, sendo nesta ocasião esclarecido o objetivo da entrevista, confirmado o número de medicamentos utilizados e marcadas a data e a hora da entrevista. Os idosos cujos prontuários eram procedentes do Posto de Saúde foram entrevistados no próprio posto, e aqueles provenientes da triagem realizada pelo Programa de Pesquisa foram entrevistados em local cedido pela Prefeitura Municipal de Gravataí.

Nas entrevistas individuais, após breve apresentação, deu-se a leitura do Termo de Consentimento Livre e Esclarecido, totalizando 100\% dos idosos que concordaram em participar do estudo. Dando seqüência, foi preenchida pela pesquisadora a ficha sócio-demográfica, o perfil farmacoterapêutico, o inventário para independência das atividades da vida diária, o auto-relato 
da adesão à prescrição médica, e então aplicado o questionário de memória prospectiva para medicamentos sob a forma de uma entrevista dirigida. Para o registro dos resultados do PMMQ foi elaborado um protocolo individual de respostas, que continha o número de identificação do idoso e as suas opções de escolha para cada questão. As entrevistas individuais tiveram um tempo médio de quarenta minutos.

\section{Resultados e Discussão}

A descrição da amostra assim como os dados referentes às estratégias de memória observados no presente estudo encontram-se a seguir.

\subsection{Descrição da Amostra}

O presente estudo incluiu 53 idosos com média de idade de 65,74 anos $(\mathrm{DP}=2,99)$, sendo que as mulheres perfaziam um total de $84,9 \%$ da amostra. A média de idade ficou situada em 65,58 anos $(\mathrm{DP}=3,08)$ para as mulheres e $66,63(\mathrm{DP}=2,45)$ para os homens. Quanto à escolaridade, 52,8\% dos idosos possuíam primeiro grau incompleto, $34 \%$ primeiro grau completo e $13,2 \%$ a partir de segundo grau incompleto até terceiro grau completo.

Os dados do presente estudo indicaram que a maioria dos idosos entrevistados mora com seus familiares, representando 90,6\% da amostra pesquisada. O que está de acordo com os dados do Instituto Brasileiro de Geografia e Estatística (IBGE) a partir de registros da Pesquisa Nacional por Amostra de Domicílios de 1995 a 1997 (RIO GRANDE DO SUL, 1997).

Segundo dados disponibilizados pelos locais em que foi recrutada a amostra, os participantes provenientes do Posto de Saúde se caracterizavam por um perfil sócio-econômico médio-baixo, e os idosos procedentes do Programa de Pesquisa eram todos residentes em uma localidade caracterizada por um perfil sócio-econômico baixo.

Ao avaliar a adesão à prescrição médica, 73,6\% dos pacientes entrevistados consideram-se aderentes, apoiando pesquisas prévias que referem o fato da superestimação da adesão quando empregado o instrumento de auto-relato (MOSCATI; PERSANO; CASTRO, 2000).

O perfil farmacoterapêutico levantou o número de medicamentos de uso contínuo e as classes terapêuticas utilizadas, e ainda a forma de aquisição dos seus medicamentos. Com relação ao número de fármacos prescritos, foi observada uma média de 4,28 medicamentos por pessoa, o que caracteriza um tratamento complexo. Os medicamentos foram listados e classificados 
de acordo com o Anatomical Therapeutic Chemical Classification System (ATCC). A classe mais utilizada pelos entrevistados foi a dos medicamentos que atuam sobre o sistema cardiovascular, seguidos daqueles empregados para o trato alimentar e metabolismo e para o sistema nervoso, cujas freqüências encontradas foram de $86,8 \%, 45,3 \%$ e 43,3\%, respectivamente. Estes dados indicam que os pacientes avaliados fazem uso de terapias que necessitam de medicamentos de uso contínuo, especificamente no caso dos fármacos administrados para doenças do sistema cardiovascular.

Quanto à forma de aquisição dos medicamentos pelos idosos entrevistados, 34\% adquiriam seus medicamentos com recursos próprios, 9,4\% recebiam no Posto de Saúde, 52,8\% compravam e retiravam via órgão público e $3,8 \%$ dos idosos recebiam por meio de um suporte familiar.

\subsection{Estratégias de Memória Utilizadas}

Foram consideradas como estratégias mais utilizadas aquelas que obtiveram média superior a 1,5, considerando uma escala de 0 a 3 , o que significa que utilizavam aquela estratégia sempre ou quase sempre no seu cotidiano. Já as menos utilizadas foram aquelas que apresentaram média igual ou inferior ao escore 1,5 , ou seja, que nunca ou quase nunca utilizavam aquela determinada estratégia. A consistência interna do instrumento de estratégias de memória adaptado, calculado pelo coeficiente de Cronbach, foi de $\alpha=0,68$, o que indica boa fidedignidade.

As freqüências do tipo de estratégias de memória utilizadas por idosos foram levantadas, e os percentuais encontrados foram de $34 \%$ para as retrospectivas e 3,8\% para as prospectivas. No que se refere à natureza das estratégias, os valores percentuais observados foram de $28,3 \%$ para as internas e $1,9 \%$ para as externas. No entanto, ao avaliarem-se as freqüências de utilização associando tipo e natureza, os percentuais encontrados foram de $66 \%$ para estratégias internas retrospectivas, $28,3 \%$ para estratégias internas prospectivas, $3,8 \%$ para as externas retrospectivas e $1,9 \%$ para as externas prospectivas. Quanto a suas médias de utilização, os resultados encontrados estão apresentados na Tabela 1. Estes dados denotam uma maior utilização das estratégias de natureza interna, independente do tipo retrospectivo ou prospectivo. 
Tabela 1 - Média do Uso de Estratégias de Memória por Natureza e Tipo. Porto Alegre, jun. 2000.

\begin{tabular}{|c|c|c|c|}
\hline \multirow{2}{*}{ Estratégia } & Retrospectiva & Prospectiva & Total \\
\hline & M (DP) & M (DP) & M (DP) \\
\hline Interna & $1,84(0,55)$ & $1,52(0,46)$ & $1,32(0,61)$ \\
\hline Externa & $0,82(0,74)$ & $0,60(0,37)$ & $0,58(0,38)$ \\
\hline Total & $1,35(0,47)$ & $0,89(0,37)$ & - \\
\hline
\end{tabular}

Notas: $\mathrm{M}=$ média; $\mathrm{DP}=$ desvio padrão.

Com relação ao tipo de estratégia, retrospectivo e prospectivo, o presente estudo encontrou uma maior freqüência de uso para as estratégias retrospectivas, concordando novamente com os achados de Gould, McDonald-Misczack e King (1997), que encontraram a média de 2,3 (DP 0,72 ) para as estratégias internas e de 0,74 (DP 0,50) para as externas. $\mathrm{O}$ maior percentual de utilização de estratégias retrospectivas pelos idosos pode estar ligado às dificuldades que eles geralmente vivenciam com relação às tarefas prospectivas, pois, conforme postulam Park e Hall (2000) e West e Craik (2001), os idosos tendem a apresentar prejuízos mnemônicos na performance prospectiva de tarefas complexas, pelo fato de as mesmas serem menos automáticas e requererem maior empenho cognitivo.

Analisando-se as respostas obtidas para cada item do questionário, foi possível identificar quais as estratégias usadas sempre ou quase sempre por no mínimo $50 \%$ dos idosos entrevistados. Em termos de estratégias externas prospectivas, somente a estratégia de deixar as embalagens em locais visíveis foi empregada sempre por mais da metade da amostra $(71,7 \%)$. Nenhuma das estratégias externas retrospectivas foi relatada como usada freqüentemente (quase sempre ou sempre) pelos idosos. Já em termos de estratégias internas prospectivas, observou-se que duas delas são sempre utilizadas pela maioria da amostra: a) no início do dia, pensar no horário dos medicamentos de modo a incluí-los no planejamento das atividades diárias (56,6\%); e b) associar o horário de auto-administrar o medicamento a algo que faça rotineiramente (75,5\%). Contudo, nenhum idoso relatou usar freqüentemente qualquer outra estratégia interna prospectiva. As estratégias mais comumente utilizadas foram as internas retrospectivas, tendo sido encontrados os seguintes resultados para idosos que relataram usá-las sempre: a) concentrar-se bem ao ler instruções sobre o uso dos medicamentos $(79,2 \%) ; b)$ concentrar-se bem quando o médico ou farmacêutico estão dando 
orientações sobre o uso do medicamento $(81,1 \%)$; e c) ler mais lentamente as instruções escritas sobre o uso dos medicamentos (54,7\%).

O PMMQ ainda oportunizou aos participantes o relato espontâneo de alguma estratégia mnemônica por eles utilizada e não incluída no questionário, perfazendo um total de $13,2 \%$ de idosos. Dentre as estratégias levantadas, todas foram externas, sendo duas retrospectivas e uma prospectiva. As retrospectivas identificadas foram: a) pedir para outra pessoa escrever na bula as informações médicas; e b) escrever na embalagem o modo de usar, ambas sendo utilizadas por $1,9 \%$ dos idosos entrevistados. A estratégia prospectiva relatada foi colocar dentro de um recipiente todos os medicamentos que irá usar durante o dia, a qual foi utilizada por $9,5 \%$ dos idosos.

Muitos idosos, pela dificuldade em aceitar as limitações inerentes ao processo de envelhecimento, como a diminuição em sua capacidade cognitiva, podem manter o uso de estratégias internas, ao invés de empregarem estratégias externas que possam ser mais efetivas para seu posterior recordar, como, por exemplo, quando rejeitam o auxílio de outros para lembrá-los de administrar seus medicamentos. No presente estudo, isto ficou evidenciado quando, por exemplo, ao responderem determinadas questões do PMMQ, os idosos diziam: "não preciso que me ajudem, eu mesmo faço". No entanto, contradiziam-se quando confirmavam que às vezes não lembravam do uso de algum medicamento.

A utilização de estratégias externas foi evidenciada nas respostas à questão aberta do PMMQ. As respostas que envolviam uma terceira pessoa referiam-se ao fato de, por exemplo, solicitar para alguém escrever na bula as informações médicas e escrever na embalagem do medicamento o modo de usá-lo, conforme o que foi também observado por Spiers e Kutzik (1995), em um estudo sobre emprego de estratégias de memória e adesão à prescrição médica. Os autores sugeriram que o baixo percentual encontrado para relato espontâneo de estratégias de memória talvez decorra da falta de entendimento por parte dos sujeitos sobre o que é considerado uma estratégia, ou seja, não percebem que as coisas que fazem para lembrar de usar seus medicamentos sejam estratégias de memória.

\subsection{Estratégias de Memória e Variáveis Sócio-Demográficas e Tera- pêuticas}

O uso de estratégias de memória, categorizadas pela natureza interna ou externa e tipo retrospectivo e prospectivo, foi avaliado no que tange às características sócio-demográficas da amostra (nível de instrução e com 
quem reside), e encontra-se descrito na Tabela 2. Na tabela, não se apresentam os dados percentuais relativos aos idosos que não utilizam essas estratégias, motivo pelo qual o total de participantes indicados difere do total da amostra.

Tabela 2 - Percentual de uso de estratégias de memória por variáveis sócio-demográficas. Porto Alegre, jun. 2000.

\begin{tabular}{lcccccccccc}
\hline \multirow{2}{*}{ Variáveis } & \multicolumn{1}{c}{ Internas } & \multicolumn{1}{c}{ Externas } & \multicolumn{2}{c}{ Retrospectivas } & Prospectivas & Total \\
\cline { 2 - 10 } & $\mathbf{N}$ & $\mathbf{\%}$ & $\mathbf{N}$ & $\mathbf{\%}$ & $\mathbf{N}$ & $\mathbf{\%}$ & $\mathbf{N}$ & $\mathbf{\%}$ & $\mathbf{N}$ \\
\hline Grau de instrução & & & & & & & & & \\
$1^{\circ}$ grau incompleto & 9 & 32,1 & 1 & 3,6 & 9 & 32,1 & 2 & 7,1 & 21 \\
$1^{\circ}$ grau completo & 5 & 27,8 & - & - & 8 & 44,4 & - & - & 13 \\
$\quad \begin{array}{c}\text { A partir de 2 } \\
\text { incompleto }\end{array}$ & 1 & 14,3 & - & - & 1 & 14,3 & - & - & 2 \\
Com quem reside & & & & & & & & & \\
$\quad$ Só & 1 & 20,0 & - & - & 1 & 20,0 & 1 & 20,0 & 3 \\
Com familiares & 14 & 29,2 & 1 & 2,1 & 17 & 35,4 & 1 & 2,1 & 33 \\
\hline
\end{tabular}

De acordo com a Tabela 2, os dados que se destacaram em termos percentuais para a amostra pesquisada indicaram que houve uma tendência a um maior uso de estratégias internas retrospectivas para os idosos com menor nível de escolaridade e que moram com seus familiares.

O emprego das estratégias de memória pelos idosos, tendo em vista as variáveis consideradas como da terapêutica - quais sejam, número de medicamentos de uso contínuo, classes terapêuticas empregadas, tempo decorrido desde a última consulta médica -, foi avaliado e encontra-se na Tabela 3, excluindo-se os participantes que não utilizavam as estratégias pesquisadas.

Tabela 3 - Percentual de Uso de Estratégias de Memória por Variáveis da Terapêutica. Porto Alegre, jun. 2000.

\begin{tabular}{lccccccccc}
\hline \multirow{2}{*}{ Variáveis } & \multicolumn{1}{c}{ Internas } & \multicolumn{1}{c}{ Externas } & \multicolumn{1}{c}{ Retrospectivas } & Prospectivas & Total \\
\cline { 2 - 10 } & $\mathbf{N}$ & $\mathbf{\%}$ & $\mathbf{N}$ & $\mathbf{\%}$ & $\mathbf{N}$ & $\mathbf{\%}$ & $\mathbf{N}$ & $\mathbf{\%}$ & $\mathbf{N}$ \\
\hline $\mathbf{N}^{\mathbf{0}}$ de Medicamentos & & & & & & & & & \\
$\quad$ Até três & 7 & 31,8 & - & - & 7 & 31,8 & 2 & 9,1 & 16 \\
A partir de três & 8 & 25,8 & 1 & 3,2 & 11 & 35,5 & - & - & 19 \\
A partir de seis & 11 & 35,5 & 1 & 3,2 & 13 & 41,9 & 1 & 3,2 & 31
\end{tabular}




\begin{tabular}{lllllllllc} 
Tempo da consulta & & & & & & & & & \\
\multicolumn{1}{l}{ Acima de 1 ano } & 1 & 20,0 & - & - & 3 & 60,0 & 1 & 20,0 & 5 \\
De 3 meses a 1 ano & 4 & 50,0 & - & - & 3 & 30,0 & 1 & 10,0 & 8 \\
1 mês até 2 meses & 4 & 25,0 & - & - & 6 & 37,5 & - & - & 10 \\
Menos de 1 mês & 6 & 35,3 & 1 & 5,9 & 6 & 35,3 & 2 & 3,8 & 15 \\
\hline Total & $\mathbf{4 1}$ & $\mathbf{3}$ & & $\mathbf{4 9}$ & & $\mathbf{7}$ & - \\
\hline
\end{tabular}

Em termos de intervalo de tempo decorrido desde a última consulta até o momento da entrevista, destacou-se o uso de estratégias retrospectivas, para aqueles que tinham comparecido ao médico há mais tempo. Segundo Einstein et al. (1998), a memória prospectiva, para tarefas rotineiras em idosos, tem um grande potencial para criar confusões em relação a ações que foram ou não executadas. Em várias questões do presente estudo, os entrevistados respondiam que tomar seu medicamento era algo que se tornara automático, que faziam sem precisar pensar. Ao reforçar os achados de McDaniel e Einsten (2000), os dados do presente estudo sugerem que, no que se refere a medicamentos de uso contínuo, aqueles cuja administração já faz parte da rotina das pessoas, o hábito transforma a tarefa em algo automático. No entanto, isto não garante que a mesma seja recordada com efetividade, pois determinadas estratégias têm sua eficácia diminuída com o passar do tempo e o avanço da idade (GOULD et al., 1997).

Além disso, Gould, McDonald-Misczack e King (1997) observaram que, quando os idosos têm muita experiência com uma determinada tarefa, como no caso do uso diário de medicamentos, tendem a adotar estratégias que correspondam às suas vivências. E, quando relatam não serem hábeis para lembrar de algo, há uma tendência para utilizarem estratégias externas, o que é positivo, pois buscam recursos como uma forma de compensar a dificuldade encontrada.

No que concerne à complexidade dos tratamentos dos idosos pesquisados, a média encontrada, de 4,28 medicamentos por pessoa, pode ser um agravante na utilização correta de seus medicamentos, e justifica o emprego de estratégias de memória pelos pacientes entrevistados. Ao comparar este resultado com o tipo de estratégia de memória empregado, os idosos que relataram maior uso de estratégias internas tinham tratamentos menos complexos, e aqueles que relataram maior uso de estratégias retrospectivas possuíam tratamentos com um maior número de medicamentos (Tabela 3). 


\section{Considerações Finais}

Neste estudo foi enfocada a freqüência do emprego de estratégias de memória por idosos, segundo diferentes dimensões sócio-demográficas e terapêuticas. Ainda que pesquisas anteriores tenham mostrado que os idosos geralmente utilizam estratégias externas com maior freqüência do que as internas em diversas situações cotidianas, vários autores (SPIERS; KUTZIK, 1995; GOULD; McDONALD-MISCZACK; KING, 1997; BRANIN, 2001; McDONALD-MISZCZAK et al., 2004) mostraram que, no âmbito específico do uso de medicamentos, os idosos utilizavam mais as estratégias de natureza interna.

Os resultados do presente trabalho parecem indicar que existe uma tendência dos idosos a utilizarem mais estratégias de memória do tipo retrospectivo do que prospectivo, corroborando os achados apresentados por Gould, McDonald-Misczack e King (1997) e Mcdonald-Miszczak et al. (2004). Considerando que a memória prospectiva envolve a recuperação de uma intenção de ação que está armazenada na memória de longo prazo (PARK; HALL, 2000), e que esta é dificultada em situações de tarefas complexas, ainda que não tenha sido realizado nenhum teste de função cognitiva neste estudo, pode-se hipotetizar que muitos dos idosos pesquisados possuíam dificuldades com a retenção da informação na memória de longo prazo.

Apesar de estes dados serem restritos à amostra pesquisada, poderão ser aprofundados em estudos posteriores no sentido de testar as diferenças observadas entre a freqüência de utilização de estratégias de memória por idosos para lembrar do uso de seus medicamentos, segundo variáveis sóciodemográficas e variáveis relacionadas à terapêutica.

Por fim, tendo em vista o comprovado declínio cognitivo de pessoas da terceira idade, estes pacientes são fortes candidatos à não-adesão à prescrição médica. São freqüentemente acometidos por doenças crônicas que requerem a administração de múltiplos fármacos, regimes terapêuticos complexos e difíceis de serem seguidos. Além disso, esta dificuldade também é reforçada pela necessidade de usarem seus medicamentos diariamente, nos mesmos horários; uma vez que a tarefa rotineira requer uma atividade mnemônica prospectiva, e esta, em idosos, encontra-se prejudicada. No caso do uso dos medicamentos, o paciente fica em dúvida se administrou ou não, ao lembrar-se da ação realizada no dia anterior. Neste sentido, o emprego de estratégias de memória eficazes, que auxiliem na recuperação de informações complexas e rotineiras, é de suma importância para evitar confusões e garantir o seguimento do regime farmacológico. No presente 
estudo, observou-se um maior uso de estratégias de memória internas, as quais são mais suscetíveis a falhas, em função do próprio processo de envelhecimento. Portanto, a partir dos resultados encontrados, pode-se sugerir que profissionais da saúde orientem e motivem os pacientes idosos para o emprego de estratégias de memória externas, intervindo positivamente na prevenção do agravamento de doenças, assim como na promoção da qualidade de vida destes pacientes e na redução de custos relacionados à saúde.

\section{MEMORY STRATEGIES USED BY ELDERLY TO REMEMBER OF THEIR MEDICATIONS}

\section{Abstract}

The multiple treatments needed to deal with diseases related to ageing make prescriptions to elderly patients normally complex and difficult to be followed. This can be even worsen by the common cognitive decline observed on people at this age, forgetfulness being one of the main reasons why they are strong candidates to non-adhesion to medical prescription. Recent studies have been showing that the use of prospective memory strategies by eldery patients can contribute for a wider therapeutic adherence. This study aimed to identifying the memory strategies used by elderly to remind of their medications. The sample was 53 non-institutionalized elderly between sixty and seventy years old, with independency to perform their daily tasks, and which used at least three prescription drugs continuously. A questionnaire, for identification of the memory strategies used by them to remind about their medication, was adapted to Portuguese and to the Brazilian reality. The adherence to the medical prescriptions was assessed by self-report. The instruments were applied to the elderly through individual structured interview. The results indicated a high level of internal retrospective strategies among the elderly interviewed, as well as a high level (73.6\%) of selfreported adherence, that reinforces previous findings about the tendency of elderly to overestimate their adhesion to medical prescription. The use of memory strategies can minimize or compensate the mnemonic difficulties observed in the elderly, such the ones to follow a therapeutic regimen, and help to preserve their autonomy and quality of life.

Keywords: Prospective memory. Memory strategies. Adherence prescription. Elderly. 


\section{REFERÊNCIAS}

BRANIN, Joan. The Role of Memory Strategies in Medication Adherence Among the Elderly. Home Health Care Services Quarterly, New York, v. 20, n. 2, p. 1-16, 2001.

BRASIL. Ministério da Saúde. Conselho Nacional de Saúde. Resolução 196, de 10 de Outubro de 1996. Diretrizes e Normas Regulamentadoras de Pesquisa Envolvendo Seres Humanos. Brasília (DF); 1996.

BROWN, Scott; PARK, Denise. Roles of Age and Familiarity in Learning Health Information. Educational Gerontology, Washington, v. 28, n. 8, p. $695-710,2002$.

CHEN, Yen-Fu; DEWEY, Michael; AVERY, Anthony. Self-Reported Medication Use for Older People in England and Wales. Journal of Clinical Pharmacy and Therapeutics, Oxford, v. 26, n. 2, p. 129-140, 2001.

CIPOLLE, Robert; STRAND, Linda; MORLEY, Peter. Pharmaceutical Care Practice: the Clinician's Guide. 2. ed. New York: McGraw-Hill, 2004.

CULOS-REED, Nicole et al. Predictors of Adherence to Behavior Change Interventions in the Elderly. Controlled Clinical Trials, New York, v. 21, n. 5, p. 200S-205S, 2000. Supplement.

EINSTEIN, Gilles; McDANIEL, Mark. Retrieval Processes in Prospective Memory: theoretical approaches and some new empirical findings. In: BRANDIMONTE, Maria; EINSTEIN, Gilles; McDANIEL, Mark. Prospective Memory: theory and applications. Mahwah: LEA, 1996. p. 115-141.

EINSTEIN, Gilles et al. Aging and Prospective Memory: the influence of increased task demands at encoding and retrieval. Psychology and Aging, Arlington, v. 12, n. 3, p. 479-488, 1997.

EINSTEIN, Gilles et al. Habitual Prospective Memory and Aging: remembering intentions and forgetting actions. Psychological Science, New York, v. 9, n. 4, p. 284-288, 1998. 
EINSTEIN, Gilles et al. Multiple Processes in Prospective Memory Retrieval: factors determining monitoring versus spontaneous retrieval. Journal of Experimental Psychology: General, Washington, v. 134, n. 3, p. 327-342, 2005.

FLORES, Liziane; MENGUE, Sotero. Uso de Medicamentos por Idosos em Região do Sul do Brasil. Revista de Saúde Pública, São Paulo, v. 39, n. 6, p. 924-929, 2005.

GENUA, Maria I. et al. Geriatria. In. FALGAS, Bonal. Farmácia Hospitalaria. Madrid: SEFH, 2002. p. 959-992.

GOULD, Odette; DIXON, Roger. Recall of Medication Instructions by Young and Elderly Adult Women. Journal of Language and Social Psychology, Clevedon, v. 16, n. 1, p. 50-69, 1997.

GOULD, Odette; McDONALD-MISCZACK, Leslie.; KING, Brenda. Metacognition and Medication Adherence: how do older adults remember? EXperimental Aging Research, Bristol, v. 23, p. 315-342, 1997.

GUYNN, Melissa; McDANIEL, Mark; EINSTEIN, Gilles. Prospective Memory: when reminders fail. Memory \& Cognition, Austin, v. 26, n. 2, p. 287-298, 1998.

HICKS, Jason; MARSH, Richard; COOK, Gabriel. Task Interference in TimeBased, Event-Based, and Dual Intention Prospective Memory Conditions. Journal of Memory and Language, New York, v. 53, p. 430-444, 2005.

JECKEL, Emilio; CUNHA, Gilson. Teorias Biológicas do Envelhecimento. In: VIANNA, Elizabete. et al. Tratado de Geriatria e Gerontologia. Rio de Janeiro: Guanabara Koogan, 2002. P. 13-19.

KATZ et al. Studies Illness in the Aged: the Index of ADL: a stardard measure of biological and psychosocial function. JAMA, Chicago, v. 185, p. 914-919, 1963.

LEITE, Silvana; VASCONCELLOS, Maria. Adesão à Terapêutica Medicamentosa: elementos para discussão de conceitos e pressupostos adotados na 
literatura. Ciência \& Saúde Coletiva, Rio de Janeiro,v. 8, n. 3, p. 775-782, 2003.

LOWRY, Kathryn et al. Intentional and Unintentional Nonadherence to Antihypertensive Medication. The Annals of Pharmacotherapy, Cincinnati, v. 39, n. 7/8, p. 1198-1203, 2005.

McDANIEL, Mark; EINSTEIN, Gilles. Strategic and Automatic Processes in Prospective Memory Retrieval: a multiprocess framework. Applied Cognitive Psychology, Chichester, v. 14, n. 7, p. 127-144, 2000.

McDONALD-MISZCZAK, Leslie et al. A Pilot Study Examining Older Adults' Beliefs Related to Medication Adherence. Journal of Aging and Health, Newbury Park, v. 16, n. 5, p. 591-614, 2004.

MORISKY, Donald; GREEN, Lawrence; LEVINE, David. Concurrent and Predictive Validity of a Self-Reported Measure of Medication Adherence. Medical Care, Philadelphia, v. 24, n. 1, p. 67-74, 1986.

MOSCATI, Iva; PERSANO, Sagramor; CASTRO, Lia Lusitana. Aspectos Metodológicos da Adesão à Terapêutica. In: CASTRO, Lia Lusitana. (Coord.). Fundamentos de Farmacoepidemiologia. Campo Grande: Grupuram, 2000. p. 170-180.

PAPALÉO-NETTO, Matheus. O Estudo da Velhice: histórico, definição do campo e termos básicos. In: VIANNA, Elizabete et al. Tratado de Geriatria e Gerontologia. Rio de Janeiro: Guanabara Koogan, 2002. p. 2-12.

PARK, Denise; HALL, Gutchess. Cognitive Aging in Everyday Life. In: PARK, Denise; SCHWARZ, Norbert. (Ed.). Cognitive Aging: a primer. Philadelphia: Psychology, 2000. p. 217-232.

PARK, Denise; KIDDER, Daniel. Prospective Memory and Medication Adherence. In: BRANDIMONTE, Maria; EINSTEIN, Gilles; McDANIEL, Mark. Prospective Memory: theory and aplications. Mahwah: LEA, 1996. P. 369-388.

PARK, Denise; MAYHORN, Christopher. Remembering to Take Medications: the importance of nonmemory variables. In: HERRMANN, Douglas 
et al. Research on practical aspects of memory. Hillsdale: Erlbaum, 2000. P. 178-289.

PARK, Denise et al. Medication Adherence Behaviors in Older Adults: effects on external cognitive supports. Psychology and Aging, Arlington, v. 7, n. 2, p. 252-256, 1992.

PAULÓS, Claudio et al. Impact of a Pharmaceutical Care Program in a Community Pharmacy on Patients with Dyslipidemia. The Annals of Pharmacotherapy, Cincinnati, v. 39, n. 5, p. 939-943, 2005.

RIO GRANDE DO SUL. Conselho Estadual do Idoso. Os Idosos do Rio Grande do Sul: estudo multidimensional de suas condições de vida. Porto Alegre, 1997.

ROTH, Mary; IVEY, Jena. Self-Reported Medication Use in CommunityResiding Older Adults: a pilot study. American Journal of Geriatric Pharmacotherapy, Hillsborough, v. 3, n. 3, p. 196-204, 2005.

ROZENFELD, Suely. Prevalência, Fatores Associados e Mau Uso de Medicamentos entre Idosos: uma revisão. Cadernos de Saúde Pública, Rio de Janeiro, v. 19, n. 3, p. 712-724, 2003.

SMITH, Rebekah. The Cost of Remembering to Remember in Event-Based Prospective Memory: investigating the capacity demands of delayed intention performance. Journal of Experimental Psychology: Learning, Memory and Cognition, Washington, v. 29, n. 3, p. 347-361, 2003.

SPIERS, Mary; KUTZIK, David. Self-Reported Memory of Medication Use by the Elderly. American Journal Health-System Pharmacy, Bethesda, v. 52, n. 9 , p. 985-990, 1995.

WEST, Robert; CRAIK, Fergus. Influences on the Efficiency of Prospective Memory in Younger and Older Adults. Psychology and Aging, Arlington, v. 16, n. 4, p. 682-696, 2001.

WORLD HEALTH ORGANIZATION. Adherence to Long-Term Therapies: evidence for action. Geneva, 2001. 


\section{APÊNDICE - Questionário Adaptado de Memória Prospectiva para Medicamentos (PMMQ)}

1) Quando o sr(a) quer lembrar do nome de um certo remédio, pede para outra pessoa ajudá-lo a lembrar? (E-R)

2) Quando o sr(a) tenta aprender os nomes dos seus remédios, o $\operatorname{sr}(a)$ tenta juntar com o nome das doenças que está tratando? (I-R)

3) O sr(a) deixa bilhetes para si mesmo em lugares fáceis de ver para lembrálo das explicações sobre seus remédios (p.ex: quantidade, como tomar, o que evitar)? (E-R)

4) $\mathrm{O}$ sr(a) repete regularmente para si mesmo as explicações de como tomar o remédio que vem utilizando há muito tempo? (I-R)

5) O sr(a) deixa as caixinhas dos remédios em lugares visíveis para lembrálo de tomar os seus remédios? (E-P)

6) Outras pessoas lhe lembram das quantidades e como os seus remédios devem ser tomados (por exemplo: tomar 1 cp.com um copo de água após as refeições)? (E-R)

7) Outras pessoas lhe lembram a que horas seus remédios devem ser tomados? (p.ex: se o sr (a) precisa tomar um certo remédio hoje à noite, alguém lhe avisa quando está na hora de tomá-lo?) (E-P)

8) O sr(a) tenta se concentrar e presta bem atenção quando está lendo explicações sobre o uso dos seus remédios (p.ex: bulas, instruções médicas escritas)? (I-R)

9) Para lembrar a hora de tomar os seus remédios, o sr(a) tenta juntar os horários a alguma coisa que o sr(a) faz todo dia (como hora do almoço, jantar, escovar os dentes)? (I-P)

10) Quando o sr(a) está recebendo explicações sobre quando e como tomar seus remédios, sr(a) imagina mentalmente esta situação (por exemplo: fica imaginando o momento de tomar o remédio, a cor, o formato do comprimido, $\mathrm{o}$ ato de encher o copo dágua)? (I-R)

11) O sr(a) pede a seu médico para falar devagarinho quando está lhe dando instruções sobre o uso dos seus remédios? (E-R) 
12) Quando o seu médico ou farmacêutico estão lhe falando sobre seus remédios o sr(a) tenta se concentrar bastante? (I-R)

13) O sr(a) escreve avisos para lhe lembrar o momento de tomar os remédios? (o horário e dia) (E-P)

14) Quando o sr(a) quer lembrar as explicações escritas dos seus remédios (bulas, instruções escritas dos médicos) o sr(a) as lê mais de uma vez? (I-R)

15) Para lembrar o momento de tomar os seus remédios, o sr(a) tenta relacionar a hora de tomar com alguma coisa que vai acontecer exclusivamente naquele dia? (p. ex.: eu tenho que tomar o remédio depois desta entrevista) (I-P)

16) Quando o sr(a) está tentando aprender explicações para uso de um remédio que ainda não usou repete mentalmente (fica repetindo para si mesmo em pensamento) as instruções para ajudá-lo a lembrar delas? (I-R)

17) No início do dia, o sr(a) pensa quando tem que tomar seus remédios de modo a incluí-los na organização de seu dia? (I-P)

18) O sr(a) usa um sinal sonoro ou relógio com alarme para lembrar-lhe quando está na hora de tomar seus remédios? (E-P)

19) $\mathrm{O}$ sr(a) fica muito tempo pensando (se preocupando) em como fazer para lembrar de tomar seus remédios na hora certa? (I-P)

20) O sr(a) risca num calendário depois de tomar seus remédios cada dia? (E-P)

21) Quando o sr(a) quer lembrar das instruções escritas do medicamento (bulas, instruções médicas) o sr(a) lê mais devagar? (I-R)

22) $\mathrm{O}$ sr(a) tem uma lista por escrito dos horários e quantidades de medicamentos que necessita usar? (E-P)

23)Eu já lhe perguntei se o sr(a) usa ou não bilhetes ou se pede ajuda de outras pessoas para ajudá-lo a lembrar de tomar seus remédios. Existe outra coisa que o sr(a) faça que eu não tenha lhe perguntado? Se existe, quão freqüente ela é?

Recebido em: 10-10-2007

$1^{a}$ revisão: 21-12-2007

$2^{a}$ revisão: $23-06-2008$

Aceite final: 26-06-2008

Estud. interdiscip. envelhec., Porto Alegre, v. 13, n. 1, p. 95-115, 2008. 